\title{
Studies of the self-assembled growth mechanism on nanocrystalline silicon nanodots
}

\author{
Samsudi Sakrani, Imam Sumpono, Nurul Aini Tarjudin and Zulkafli Othaman \\ Ibnu Sina Institute for Fundamental Science Studies, Universiti Teknologi Malaysia, 81310 UTM Skudai Johor, Malaysia \\ Received 23 February 2010, Revised 7 July 2010, Accepted 15 July 2010, Available online 8 October 2010
}

\begin{abstract}
Nanocrystalline silicon (nc-Si) nanodots have been grown on corning glass (7059) substrate using a self-assembly VHF-PECVD method under the following experimental conditions: Fixed deposition temperatures of $300 / 400{ }^{\circ} \mathrm{C}$, deposition times $30 / 60 \mathrm{~s}$, plasma power of $10 \mathrm{~W}$, silane gas flow rate of $10 \mathrm{sccm}$, as well as deposition pressure of $10^{-2}$ torr. It is predicted that the onset of nucleation began immediately after the deposition and start to appear clearly after 20-60 s during which growth mechanisms occur. Essentially, the nanodots were formed onto the substrate in dome-like shapes by virtue of equilibrium surface energies, $\gamma_{L S}, \gamma_{L_{N}}$ and $\gamma_{N S}$. The associated liquid/solid nucleation mechanism was then simulated and related parameters were obtained: Free energy change per unit volume $\Delta G_{v} \sim-10^{4} \mathrm{Jmol}^{-1}$; Surface energies per unit area, $\gamma_{L N}=1.44 \mathrm{Jm}^{-2}, \gamma_{N S}=19-60 \mathrm{Jm}^{-2}$ and $\gamma_{L S}=0.74 \mathrm{Jm}^{-2}$; Critical energies $\Delta G^{*} \sim 10^{-15} \mathrm{~J}$; Critical radii $r^{*}=16-48 \mathrm{~nm}$. These results were experimentally verified, in particular for selected critical radius $r^{*}$ less than $50 \mathrm{~nm}$. Other measurements were also carried out: PL analysis gave bandgap energies $\sim 1.8-2.4 \mathrm{eV}$, whilst Raman spectra revealed the coexistence of nc-Si and amorphous Si. It is strongly suggested that, the nc-Si nanodot grown on glass substrate fulfills the Volmer-Weber growth mode with a minor modification.
\end{abstract}

| Nanocrystalline | Silicon | Self-Assembled Growth | Nanodot | Corning Glass |

( 2010 Ibnu Sina Institute. All rights reserved. http://dx.doi.org/10.11113/mjfas.v6n2.197

\section{INTRODUCTION}

Nanodot is an artificial nanostructure having diameters between 10 - $100 \mathrm{~nm}$ beyond which quantum dot $(<10 \mathrm{~nm})$ and thin film $(>100 \mathrm{~nm})$ are dominant. It can fill up to more than $10^{5}$ atoms, i.e. about ten times the number of atoms in a quantum dot. Recent studies on nanocrystalline silicon (nc-Si) revealed its potential applications in wide areas of interest, and it is the purpose of this paper to investigate the growth of nanodots both from the stand points of theoretical prediction and experimental evidence.

Nanocrystalline silicon is an allotropic form of silicon with paracrystalline structure, i.e., having short and medium range ordering in their lattice but lacking long range order. It has grains sizes less than $100 \mathrm{~nm}$ and normally exists in amorphous phase [1,2]. For nc-Si thin films fabricated using CVD methods varying grain structures are observed to be embedded in the amorphous matrix and its sizes are much dependent on the experimental conditions. The crystalline-amorphous changes are still the subject of interest, but generally can be envisaged as bonding rearrangement occurring in amorphous stage. nc-Si has many useful advantages over a-Si, one being that if grown properly it can have a higher electron mobility, due to the presence of the silicon crystallites. It also shows in-

Corresponding author at: Ibnu Sina Institute for Fundamental Science Studies, Universiti Teknologi Malaysia, 81310 UTM Skudai, Johor, Malaysia

E-mail addresses: samsudi2@gmail.com (Samsudi Sakrani) creased absorption in the red and infrared wavelengths, which make it an important material for use in a-Si solar cells [3]. One of the most important advantages of nanocrystalline silicon, however, is that it has increased stability over a-Si, one of the reasons being because of its lower hydrogen concentration. Although it currently cannot attain the mobility that poly-Si can, it has the advantage over poly-Si that it is easier to fabricate, as it can be deposited using conventional low temperature a-Si deposition techniques, such as PECVD, as opposed to laser annealing or high temperature CVD processes, in the case of poly-Si.

The main application of this novel material is in the field of silicon thin film solar cells. As nc-Si has about the same bandgap as crystalline silicon, which is $\sim 1.12 \mathrm{eV}$, it can be combined in thin layers with a-Si, creating a layered, multi-junction cell called a tandem cell. The top cell in a-Si absorbs the visible light and leaves the infrared part of the spectrum for the bottom cell in nanocrystalline Si $[3,4]$.

\subsection{Nucleation and growth theory}

An understanding of the growth mechanism can be dated back to the classical nucleation theory [5] and the three growth modes of nanofilm [6]. The diagram in Fig 1. distinguishes different growth mechanisms, including twodimensional (a) layer-by-layer growth or Frank-van der 
Merwe (F-M), (b) island growth or Volmer-Weber (V-W), and (c) mixed growth of Stranski-Krastanov (S-K). These growth modes generate stable islands, whereas three different kinds of ripening which may or may not include a two-dimensional layer (frequently called the wetting layer) are possible. The diagrams demonstrate that in order to achieve stable and controllable S-K growth, for which uniform small islands or nanodots may result, it is necessary to employ semiconducting crystal systems that include a large amount of strain resulting from structural misfits, $\varepsilon$ (i.e., work far out along the horizontal axis in this diagram), and the deposited material must not exceed a certain critical coverage.

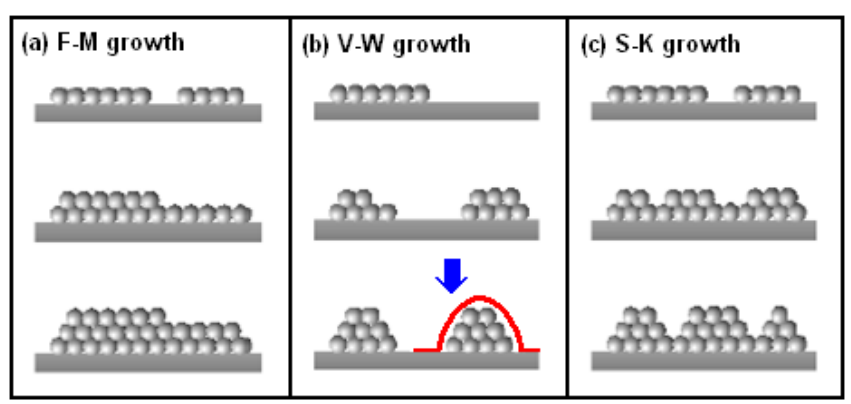

Fig. 1. Nanofilm growth modes: (a) F-M, (b) V-W and (c) S-K growth

For the case of V-W growth, the contribution of misfits at the material-substrate interface is no longer significant, except for certain semiconductors with suitable confirmation of phase diagram. There is a possibility that, $\mathrm{V}-\mathrm{W}$ growth can occur in amorphous and non-crystalline semiconductors if the surface parameters are taken into consideration. These normally refer to the classical nucleation theory where surface energies acting on the nucleus play important roles in determining equilibrium condition for the growth of clusters on the substrate. Fig. 1(b). When a liquid is transformed into a solid phase the transition is often initiated by a nucleation process, manifested by the appearance of nuclei of the new phase. The process is made possible by diffusion of atoms or molecules that aggregate to form the nuclei of liquid, with the size of these nuclei increases with time and at the end of the process a new homogenous solid is formed, i.e. 3dimensional islands with increased volume. Bauer (1980s) was the earliest to describe thin film growth in terms of surface energy with the relation: $\gamma_{\text {film }} \leq \gamma_{\text {substrate }}-\gamma_{\text {interface }}$. Under the V-W growth mode, a stable nucleus is governed by three surface energies per unit area, $\gamma_{L S}, \gamma_{L N}$ and $\gamma_{N S}$ and contact angle, $\theta$ according to the relationship:

$\gamma_{L S} \leq \gamma_{L N} \cos \theta+\gamma_{N S}$

which satisfies the condition of $-1<\cos \theta<0$ (for $\theta>90^{\circ}$ ). Accordingly, the atoms of the nucleus are bound to each other much stronger than that of the atomic substrate, and finally settled in oval shaped nanodot. This non-wetting condition occurs when the nucleus is being pulled inside (higher value of $\gamma_{L N} \cos \theta+\gamma_{N S}$ ) rather pushed backward. It appears that, the V-W's oval shape and the predicted domelike nanodot in Fig. 2 seems contradictory, but both are considered identical if the total of $\gamma_{L N} \cos \theta+\gamma_{N S}$ is just fairly greater than $\gamma_{L s}$. Such a deposition phenomena may occurs in metals/semiconductors on glass/dielectric/oxide substrates [7], and for this particular investigation the nanofilm (nanodot) of nc-Si appears to be easily adapting to corning glass substrate just like the amorphous silicon [8].

\subsection{Supercooled liquid-solid nucleation}

The classical theory of heterogeneous nucleation treats the forming nucleus as part of a sphere attached to the substrate, which is different from a spherical droplet considered for homogeneous nucleation. Fig. 1 (b) shows a typical shape of nucleus which is capped in red, known in different names such as, cap, dome, inverted bowl-shaped. This nucleus is thought to consist of incompressible, uniform liquid/solid and a part of a sphere with the base attached to the insoluble surface. There are interfaces between the solid nucleus-substrate surface, liquid-solid nucleus and liquid-substrate surface, each of which is shown by the respective surface energies per unit area, $\gamma_{N S}$, $\gamma_{L S}$, and $\gamma_{L N}$. The angle $\theta$ between the nucleus surface and the substrate surface is called the contact angle. The critical radius in heterogeneous nucleation is the same as in homogeneous nucleation, as it only depends on $\Delta G_{v}$ (change in energy of solidification). The substrate surface is usually considered homogeneous, although there is a possibility of some non-uniform surfaces. In this investigation planar substrate surface geometry is treated.

From the thermodynamic point of view, a change in the free enthalphy, $\Delta G$ is associated with nucleation via the following four processes: Firstly, creation of new volume of the nucleus (negative); secondly existence of surface area of liquid-nucleus (positive), thirdly existence of surface area of liquid-nucleus (positive) and lastly the energy lost at liquidsubstrate interface (positive). Thus [8],

$\Delta G=\beta_{3} r^{3} L_{f} \frac{\Delta T}{T_{m}}+\beta_{1} r^{2} \gamma_{L N}+\beta_{2} r^{2} \gamma_{N S}-\beta_{2} r^{2} \gamma_{L S}$

where, $\beta_{1}=2 \pi(1-\cos \theta), \beta_{2}=\pi \sin ^{2} \theta, \beta_{3}=\pi\left(2-3 \cos \theta+\cos ^{3} \theta\right.$ )$/ 3, \Delta G_{v}=L_{f}\left(\Delta T / T_{f}\right)$ is the energy change per unit area of the nucleus and has the values depending on the undercooling temperature, $\Delta T\left(T_{m}-T\right)$; positive if $T>T_{m}$, with $T_{m}$ being the temperature of the transition (solidification temperature), $L_{f}$ is entalphy of fusion. Under these conditions, applying the Gibbs-Duhem principle, the new phase is not stable. Since $\gamma$ is a positive quantity, $\Delta G_{v}$ is then positive: the number of nuclei of the new phase are not stable and do not tend to grow. On the contrary, if $T<T_{m}$, is negative, the new phase (solid) is stable. There are values of $r$ for which $\Delta G_{v}<0$, and the corresponding nuclei will tend to stabilize the new phase since their formation reduces the free enthalpy of material. When $T<T_{m}(\Delta T>0), \Delta G_{v}$ has a 
maximum for $r=r^{*}$. For $r>r^{*}$, the formation of nuclei of increasing size can results in stabilization of the solid phase since $\Delta G_{v}$ decreases and even becomes negative. Both the critical energy, $\Delta G^{*}$ and critical radius, $r^{*}$ are obtained as,

$$
\begin{aligned}
& \Delta G^{*}=\frac{16 \pi \gamma_{N S}^{3} T_{m}^{2}}{3 L_{f}^{2} \Delta T^{2}}\left(\frac{2-3 \cos \theta+\cos ^{3} \theta}{4}\right) \\
& r^{*}=\frac{-2 \gamma_{N S}}{\Delta G_{V}}=\frac{-2 \gamma_{N S} T_{m}^{2}}{L_{f}^{2} \Delta T^{2}}
\end{aligned}
$$

$\Delta G^{*}$ is equivalent to the potential barrier that must be lowered for nuclei of critical size $r^{*}$ to be formed more easily. It seems that from equation (2) the more important supercooling is, the more this barrier is lowered. The Volmer thermodynamic model shows that a complete phase transition by nucleation is only possible if the liquid is supercooled. In fact, nucleation is initiated with lower supercooling (larger $\Delta T$ ) because the impurities present in the liquid phase, even in low concentration, induce nucleation, normally referred to heterogeneous nucleation.

\section{EXPERIMENTAL}

The samples were fabricated using a home-made very high frequency $(150 \mathrm{MHz})$ plasma enhanced chemical vapour deposition system (VHF-PECVD) at a chamber pressure $10^{-2}$ Torr, substrate temperatures $300{ }^{\circ} \mathrm{C}$ and 400 ${ }^{\circ} \mathrm{C}$, fixed argon flow rate of $10 \mathrm{sccm}$, and deposition times varied between 20-60 seconds. The principal process was based on flowing a known rate of silane gas (silane10\%/argon90\%) in a specially designed chamber, and allowing $150 \mathrm{MHz}$ RF supply to effectively enhance the chemical processes whereby the silicon particles produced is buried on the substrate (corning glass) and other byproducts are released to the atmosphere. Selected samples fabricated at $300^{\circ} \mathrm{C} / 25 \mathrm{~W} / 15$ mins were analysed using photoluminescence and XRD in order to get better resolution of the spectrums. A theoretical investigation was done using the classical approach of heterogeneous nucleation in which the net energy change required to form a spherical nucleus is equivalent to the summation of energy terms associated with four different interfacial interactions.

\section{RESULTS AND DISCUSSION}

The analysis obtained from AFM (Figure 2) shows that the nanodots fabricated at $300{ }^{\circ} \mathrm{C}(573 \mathrm{~K})$ may start to form earlier, but they seem to be more defined and observable at the time of $30 \mathrm{~s}$ after the deposition. Under such a condition, less number of dots is formed on the substrate, approximately around 28 dots per $\mu \mathrm{m}^{2}$. Further analysis on the cross sectional area reveals the dot's patterns in dome-like shape, which measure around $45.11 \mathrm{~nm}$ in diameter. This appearance is contrary to a pole-like shape observed in Fig.2. For the nanodots fabricated at a temperature of $400{ }^{\circ} \mathrm{C}(673 \mathrm{~K})$ and similar deposition time (Fig. 4a), marked changes occurs in both density and size of 134 dots per $\mu \mathrm{m}^{2}$ and $\sim 88.11 \mathrm{~nm}$ (Fig. 4b), respectively. The dependence of contact angle, $\theta$ at $70^{\circ}$ was found to be less significant as can be observed for the case of $r^{*}$ in Fig. 7. Some selected samples were analyzed using photoluminescence (PL) and XRD in order to determine the bandgap energy, $E_{g}$ and contents, respectively. From PL spectrum shown in Fig. 5, $E_{g}$ were found to lie between $\sim 1.8-2.4 \mathrm{eV}$, the values which are greater than that of bulk silicon ( $>1.1 \mathrm{eV}$ ) thus confirming the existence of nanodots in the samples. This is supported by the Raman analysis for $300 / 400{ }^{\circ} \mathrm{C}$ samples (Fig. 3) which reveals the coexistence of both nc-Si crystal at $517 \mathrm{~cm}^{-1}$ and amorphous $\mathrm{Si}$ at $480 \mathrm{~cm}^{-1}$ [9]. All the above results are different from those of Si quantum dots [8] fabricated using the sputtering method.

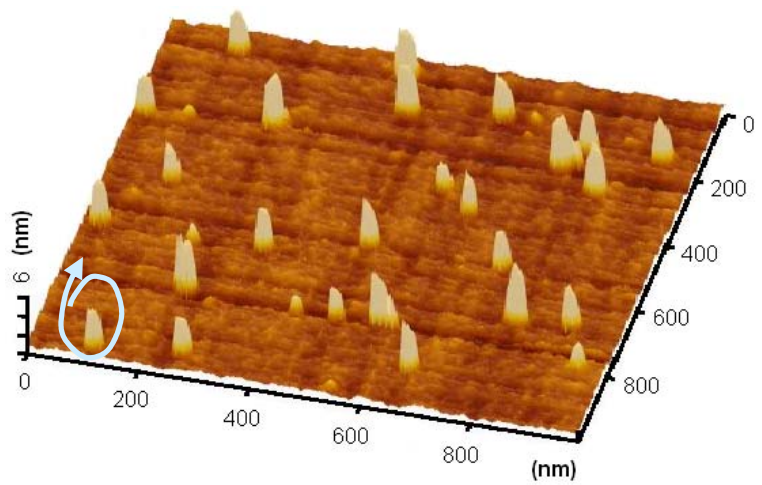

Fig. 2. Typical AFM image of nc-Si nanodots formed at $573 \mathrm{~K} / 30 \mathrm{~s} / 10 \mathrm{~W} / 10$ sccm

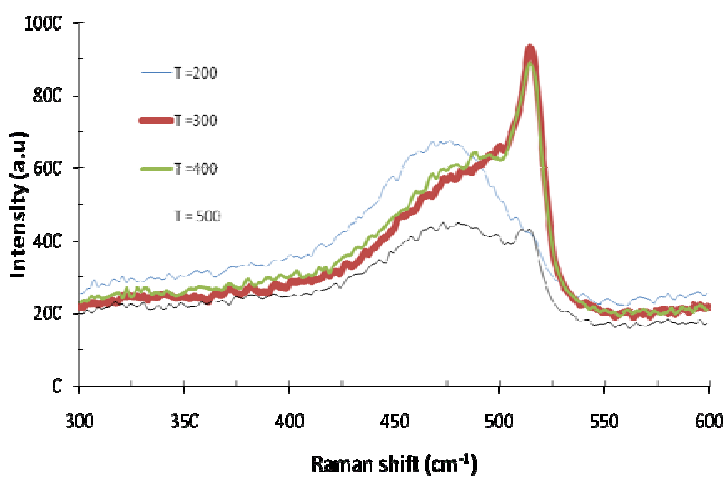

Fig. 3. Raman spectra of of nc-Si nanodots formed at different temperatures

Simulation works have been done based on the classic nucleation theory, especially equations (2) to (4). A computer program was written using visual basic to include the following parameters: Enthalpy of fusion, $L_{f}=3.96 \mathrm{x}$ $10^{4} / \mathrm{mol}$; melting temperature of silicon, $T_{m}=1683 \mathrm{~K}$; 
substrate temperature, each for $T=573 \mathrm{~K}$ and $673 \mathrm{~K}$; undercooling temperature, each for $T_{m}-T=1110 \mathrm{~K}$ dan 1010 $\mathrm{K}$ respectively, atomic volume, $v_{o}=12.1 \mathrm{~cm}^{3} / \mathrm{mol}$ and contact angle, $\theta=70^{\circ}$. The simulation results have been obtained and are shown in Table 1 (for $T=573 \mathrm{~K}$ ).

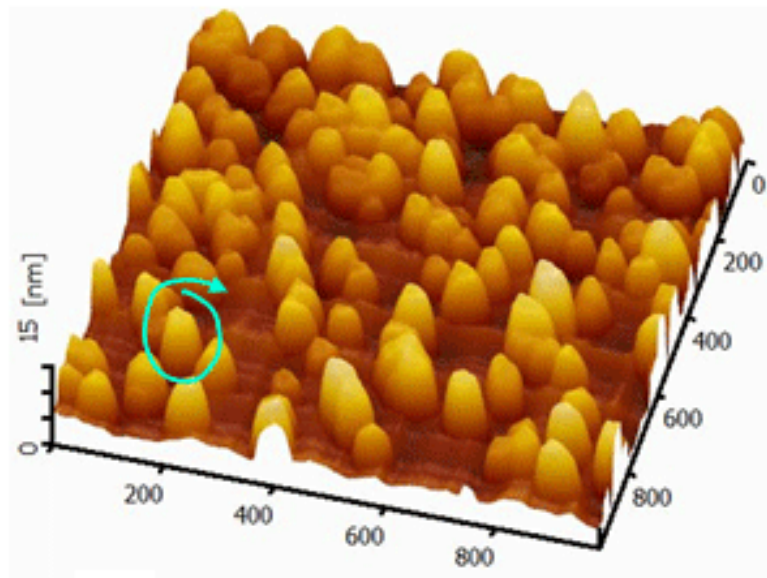

a)

a)

$[\mathrm{nm}]$
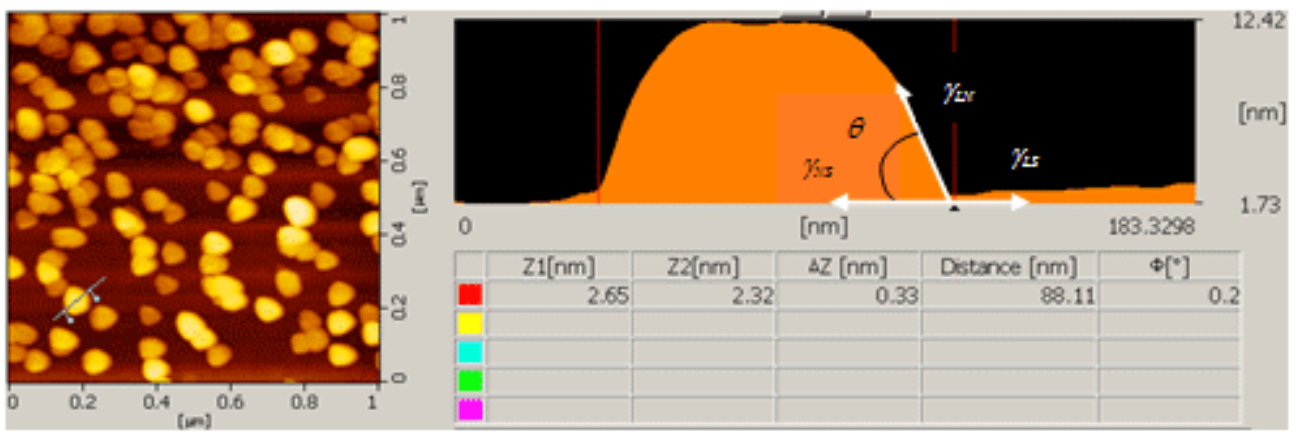

b)

Fig. 4. Typical AFM images of nc-Si nanodots a) formed at $673 \mathrm{~K} / 30 \mathrm{~s} / 10 \mathrm{~W} / 10 \mathrm{sccm}$ - circled, and b) measured density $\sim 134$ dots per $\mu \mathrm{m}^{2}$ and diameter $\sim 88.11 \mathrm{~nm}$.

Table 1. Experimental and computed growth parameters

\begin{tabular}{|c|c|c|c|c|}
\hline \multicolumn{2}{|c|}{ Experiment, } & $\mathrm{T}=573 \mathrm{~K}$ & \multicolumn{3}{|c|}{ Simulation } & \multicolumn{2}{|c|}{ Fixed, $\gamma_{L N}=1.44 \times 10^{-4} \mathrm{Jcm}^{-2}$} & $\gamma_{L S}=7.37 \times 10^{-5} \mathrm{Jcm}^{-2}$ \\
\hline$d(\mathrm{~nm})$ & $r^{*}(\mathrm{~nm})$ & $\gamma_{N S}\left(\mathrm{Jcm}^{-2}\right)$ & $r^{*}(\mathrm{~cm})$ & $\Delta G^{*}(\mathrm{~J})$ \\
\hline 32.17 & 16.09 & $19.8 \times 10^{-4}$ & $1.61 \times 10^{-6}$ & $3.71 \times 10^{-16}$ \\
\hline 45.11 & 22.56 & $27.8 \times 10^{-4}$ & $2.26 \times 10^{-6}$ & $1.02 \times 10^{-15}$ \\
\hline 54.56 & 27.28 & $33.7 \times 10^{-4}$ & $2.73 \times 10^{-6}$ & $5.37 \times 10^{-15}$ \\
\hline 78.38 & 39.19 & $48.4 \times 10^{-4}$ & $3.92 \times 10^{-6}$ & $7.63 \times 10^{-15}$ \\
\hline 88.11 & 44.06 & $54.5 \times 10^{-4}$ & $4.41 \times 10^{-6}$ & $9.58 \times 10^{-15}$ \\
\hline 95.07 & 47.54 & $58.8 \times 10^{-4}$ & $4.75 \times 10^{-6}$ & \\
\hline
\end{tabular}

Figure 6 shows the variations of $r^{*}$ and $\Delta G^{*}$ with $\gamma_{N S}$ for $T=573 \mathrm{~K}$. The graph clearly indicates an increasing mode with a linear and curvy variations in $r^{*}$ and $\Delta G^{*}$, respectively as predicted from the theory. There are also points on the graphs indicating the measured values of $r^{*}$ ranging between $16-48 \mathrm{~nm}$ corresponding to nanodot's sizes of $32-95 \mathrm{~nm}$. These results strongly suggest that both critical parameters are the functions of surface energy at the nucleus-surface interface and contact angle between the nucleus and substrate. The $\mathrm{V}-\mathrm{W}$ growth mode was found to fulfill equation (1), since the sum of $\gamma_{L N} \cos \theta+\gamma_{N S}$ is always greater than $\gamma_{L S}$. An example of simulated parameters for $T=573 \mathrm{~K}$ is tabulated in Table 1 , together with the measured diameters and $r^{*}$. Analysis results for $T=673 \mathrm{~K}$ (not shown) are generally similar in the trends of graphs but slightly different in the appearance of nanodots. It is important to note that, there is a very good compromise between the theoretical and experimental data, especially the value $\gamma_{L N}$ $=1.44 \times 10^{-4} \mathrm{Jcm}^{-2}[10,11]$. 
It is recommended that from the above discussion the self-assembled growth occurring in nc-Si nanodots is dominated by the Volmer-Weber growth mechanism. Accordingly, the oval-shaped islands are formed due to atomic binding contributed by neighbouring atoms according to a relationship given by equation (1). However, the expected growth process satisfies the classical nucleation theory on the assumption that the nucleus formed must be in a cap-like shape (part of spherical shape). The contradiction between the oval and the cap-like shape can be resolved by adapting reduced amount of atomic binding amongst the neighbouring atoms, with the original relationship remain unchanged. In other words slight modification is required in order that the sum of $\gamma_{L N} \cos \theta+$ $\gamma_{N S}$ is just reasonably greater than $\gamma_{L S}$. Experimental verification has been successfully done and found to be in a very good agreement.

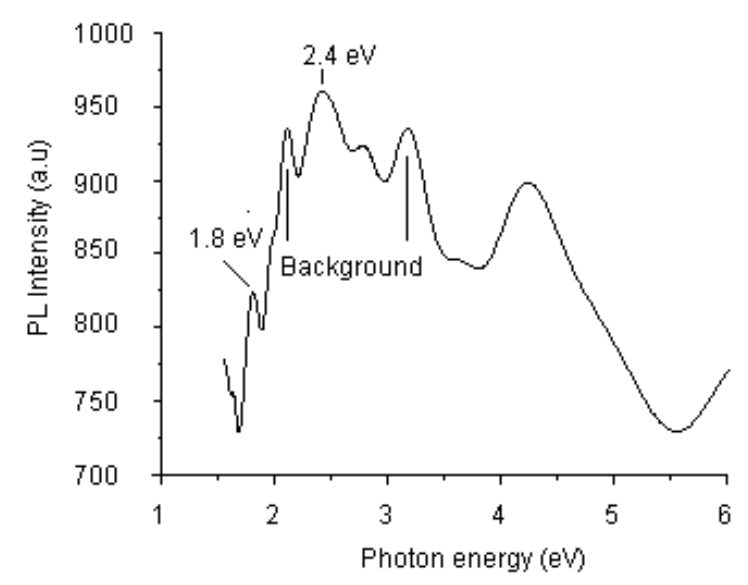

Fig. 5. PL spectrum for a selected sample ( $T=673 \mathrm{~K})$

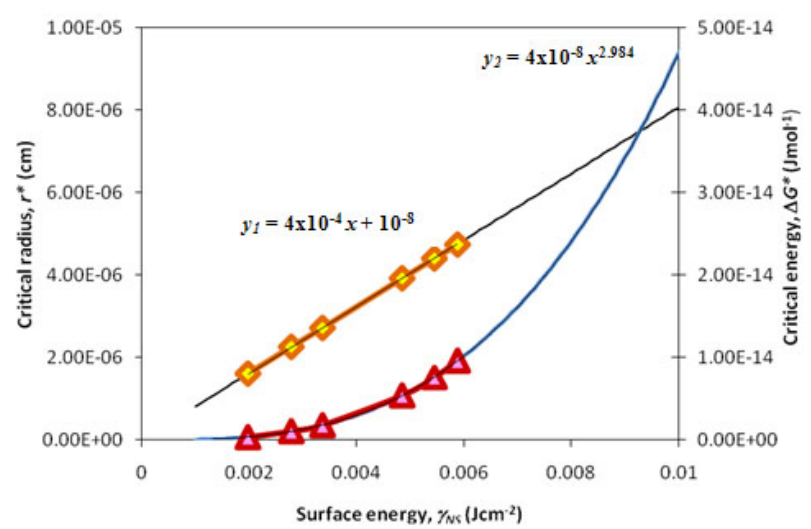

Fig. 6. Variations of $r^{*}$ and $\Delta G^{*}$ as a as function of surface energy, $\gamma_{N S}$ for $T=573 \mathrm{~K}$

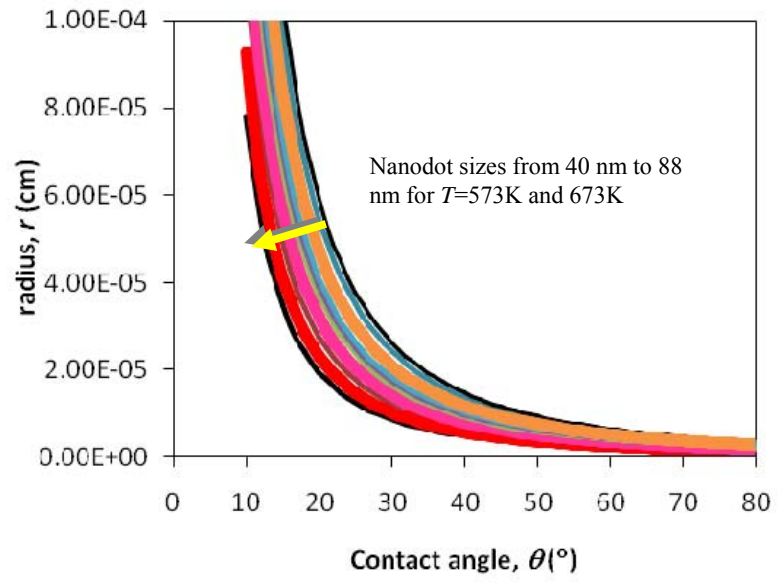

Fig. 7. Variations of $r^{*}$ and $\Delta G^{*}$ as a function of contact angle, $\theta$.

\subsection{CRESE point}

!

! There is an interesting feature that can be extracted from the plot in Fig. 6. Solving the mathematical regression the following functions are obtained for the linear and curve plots, respectively, i.e., $y_{1}\left(=r^{*}\right)=4 \times 10^{-4} x+10^{-8}$ and $y_{2}$ $\left(=\Delta G^{*}\right)=4 \times 10^{-8} x^{2.984}$. Assuming that $2.984 \sim 3.0\left(y_{2}\right.$ : experimental linear equation) and both functions are equal, i.e. $\mathrm{Y}_{1}=y_{2}$, then a cubic equation, $4 \times 10^{-8} x^{3}-4 \times 10^{-4} \times-10^{-8}$ $=0$ is formed. There are two possible solutions for surface energy $x$, namely $x_{1} \approx 10^{-2} \mathrm{jcm}^{-2}$ and $x_{2} \approx-61.6 \mathrm{jcm}^{-2}$. Except $x_{2}, x_{1}$ is an extreme point representing positive $r^{*}$ and $\Delta G^{*}$ which lies within the practical limit. It can be considered as a point of equilibrium between the critical radius-energy and surface energy or so-called CRESE point. Further analysis on different temperatures could result in different CRESE points and a new relationship could be developed. A general relationship can be established as follows:

Rewriting eqs. (3) and (4) as

$y_{1}\left(=r^{*}\right)=\mathrm{A} x^{3}$ and $y_{2}\left(=\Delta G^{*}\right)=\mathrm{B} x+\mathrm{C}$

Then, equating both equations to form a general CRESE equation,

$\mathrm{A} x^{3}-\mathrm{B} x-\mathrm{C}=0$

where

$A=\frac{16 \pi T_{m}^{2}}{3 L_{f}^{2} \Delta T^{2}}\left(\frac{2-3 \cos \theta+\cos ^{3} \theta}{4}\right)$

$B=\frac{-2 T_{m}^{2}}{L_{f}^{2} \Delta T^{2}}$

and $\mathrm{C}$ is a constant 
Equation (5) can be solved mathematically in order to determine the two CRESE points for each set of temperature.

\section{CONCLUSION}

The early stage of nanocrystalline silicon nanodot formation can be well described using the Volmer-Weber growth mode mechanism, based on the non-wetting oval shape cluster. Slight modifications of this oval to dome-like shape are needed in order to satisfy the liquid-solid nucleation theory. Accordingly, the sums of $\gamma_{L N} \cos \theta+\gamma_{N S}$ are just reasonably greater than $\gamma_{\mathrm{LS}}$, and these have been verified experimentally. The self-assembly method can be applied for the fabrication of quantum devices, such as quantum dot single-electron transistor and nanobiosensor.

\section{ACKNOWLEDGEMENT}

The authors would like to thank the Ministry of Higher Education for providing financial support under the FRGS grant, Vot No. 78461.

\section{REFERENCES}

[1] X. Zhao, O. Schoenfeld, S. Nomura, S. Komuro, Y. Aoyagi and T. Sugano, Mat. Sci. \& Eng., B35 (1995) 467-471.

[2] J. Heitmann, F. Müller, M. Zacharias, and U. Gösele, Advanced Materials, vol. 17, no. 7 (2005) 795-803.

[3] Y. Hamakawa, Thin Film Solar Cells: Next Generation Photovoltaics and Its Applications, Springer Verlag (2004).

[4] S. Tripathy, R. K. Soni, S. K. Ghoshal And K. P. Jain, Bull. Mater. Sci. (Indian Academy of Sciences), Vol. 24, No. 3 (2001) $285-289$.

[5] S. Sakrani, Q. J. Lim and Y. Wahab, J. Fundamental Sciences, 1, No.1 (2005) 21-31.

[6] Schmezer, G. Ropke, and V.B. Priezzhev Eds. Nucleation Theory and Applications, JINR, Dubna (2002).

[7] R. R. Tummala and B. J. Foster, J. Mat. Science, 10, No. 5 (1975) 1575-4803.

[8] S. Sakrani, Q. J. Lim and Y. Wahab, J. Fundamental Sciences, 3, No.1 (2007) 158-165.

[9] J. Lee, M. Shon and J. H. Lyou, Current Appl. Phys., (2006) e54-e57.

[10] D.J. Eaglesham, A.E. White, L.C. Feldman, N. Moriya, and D.C. JacobsonPhysical Review Letters., 70(11) (1993) 1643-1646.

[11] I. Satoshi, H. Shotaro and S. Shinsuke. J. of the Society of Materials Science (Japan), 52 (2003) 231-234. 